10 Mase S, Chorba T, Lobue $\mathrm{P}$, et al. Provisional CDC guidelines for the use and safety monitoring of bedaquiline fumarate (Sirturo) for the treatment of multidrug-resistant tuberculosis. MMWR 2013; 62: 1-12.

11 World Health Organization. Companion Handbook to the WHO Guidelines for the Programmatic Management of Drug-Resistant Tuberculosis. Geneva, WHO, 2014.

12 Falzon D, Jaramillo E, Schünemann HJ, et al. WHO guidelines for the programmatic management of drug-resistant tuberculosis: 2011 update. Eur Respir J 2011; 38: 516-528.

13 Pontali E, Sotgiu G, D’Ambrosio L, et al. Bedaquiline and multidrug-resistant tuberculosis: a systematic and critical analysis of the evidence. Eur Respir J 2016; 47: 394-402.

14 Aung KJM, Van Deun A, Declercq E, et al. Successful "9-month Bangladesh regimen" for multidrug-resistant tuberculosis among over 500 consecutive patients. Int J Tuberc Lung Dis 2014; 18: 1180-1187.

15 IUALTD, Inc. The Evaluation of a Standard Treatment Regimen of Anti-tuberculosis Drugs for Patients with MDR-TB (STREAM). https://clinicaltrials.gov/ct2/show/record/NCT02409290. Date last accessed: July 16, 2015. Date last updated: April 3, 2015.

\title{
An overview on tuberculosis-specific hospitals in China in 2009: results of a national survey
}

To the Editor:

As of 2014, tuberculosis (TB) was the deadliest infectious disease worldwide and China has the third-highest TB burden [1]. In that year alone there were 826155 new cases in China [1]. In addition, China has been experiencing a serious epidemic of drug-resistant TB $[2,3]$.

The availability of adequately equipped microbiology laboratories is an essential element of effective TB control $[4,5]$. The Chinese National Center for TB control (NCTB), which was organised through the public health system throughout the 1990s and early 2000s, invested in this area. As many as 3490 TB dispensaries were established [6]. In 2005, \$1.3 billion was invested to rebuild 2448 dispensaries and scale up new TB tests such as liquid culture $[7,8]$.

Since early 2000, the NCTB programme has been moving TB care towards a hospital-based management model which mandates tuberculosis-specific hospitals to diagnose and treat TB patients [8]. These hospital are public hospitals or health facilities and are designated by their local authority to clinically manage TB patient. Variants include TB hospitals, chest hospitals and infectious disease hospitals, etc. [8]. At the moment, there is little knowledge regarding the testing capacity of laboratories within these hospitals. Additionally, there is also little data about the prevalence of multi drug-resistant TB (MDR-TB) in tuberculosis-specific hospitals. The latest national MDR-TB survey in China was conducted only in TB dispensaries, i.e. within the public health system. In this study, we aim to address the aforementioned issues by conducting a national survey in microbiology laboratories in tuberculosis-specific hospitals in 2009.

The survey was conducted using a standardised questionnaire designed by the National Tuberculosis Clinical Center. We collected cross-sectional information from all 203 tuberculosis-specific hospitals in China in existence in 2009 with at least 30 beds in TB wards. The study was conducted in 31 provinces, municipalities, and autonomous regions, with questionnaires completed by directors of $\mathrm{TB} /$ respiratory department, physicians, and laboratory technicians. A training course for data collection officials was held at the National Tuberculosis Clinical Center to ensure data integrity.

The questionnaire gathered information on the usage of four TB tests, and the number of drug-resistant TB cases diagnosed. The four tests include sputum smear microscopy, solid and liquid culture, and phenotypic drug susceptibility test (DST), which are recommended by the 2008 National Guidelines [9]. Because other tests, such as nucleic acid amplification test, were not approved in China for detection of TB between 1999 and 2009, data on these tests had not been regularly collected by TB hospitals and could not be included. Testing capacity data for the 203 tuberculosis-specific hospitals was retrieved for the year 2004 and 1999. Overall test volume per hospital and average number of tests performed per hospital were recorded on an annual basis. Since these hospitals had not participated in external quality assessment until 2010, we had no 
data on the quality of the tests performed by the time of the survey. To guarantee the robust nature of the results, $20 \%$ of the questionnaires were cross-checked with original hospital records obtained from the relevant Provincial Bureau of Health. Discrepancies were resolved by telephone interviews.

Of the 203 TB-specific hospitals surveyed, 198 (98\%) had the capacity to conduct smear microscopy in 2009 (table 1), thereby indicating this was the most widely used diagnostic test. Roughly 60\% of tuberculosis-specific hospitals had the capacity to perform solid culture and/or a DST in 2009. Liquid culture, however, were much less common; only 62 hospitals (31\% of the total) were able to conduct liquid culture in 2009 as biological safety level 3 is required for the labs to conduct liquid culture [10]. Nevertheless, it should be noted that the ability to conduct liquid culture had been quickly growing. Between 1999 and 2009, the number of hospitals capable of performing this test had increased from 20 to 62, an improvement in alignment with the ambition of the China National Plan for 2006-2010 [11]. The development of TB laboratory capacity was also impressive in other respects. There were 1.2-times the number of TB-specific hospitals capable of performing microscopy smear testing in 2009 than in 1999, 1.3-times the number of such hospitals that could perform solid culture and 1.5-times the number of TB-specific hospitals that could undertake a DST.

In addition to analysing the capability of TB-specific hospitals to conduct each specific type of test, we also considered the total numbers of each test conducted in 2009. In terms of the average annual number of tests performed in each TB-specific hospital (with that particular capability), there were a slightly higher number of liquid culture $(n=1844)$ performed per hospital than solid culture $(n=1259)$. Only 428 drug susceptibility tests were performed per hospital. Overall annual test throughput per hospital also increased significantly between 1999 and 2009, especially for a DST, where there was a 1.4 times increase in the number of tests per hospital.

A total of 1304 laboratory technicians were employed in the 203 hospitals in 2009, which meant that there were, on average, 6.4 technicians at each hospital. However, there was a geographic variation in the distribution of lab technicians; the highest number was in the West (7.8 technicians per hospital), compared with a relatively lower number in the East (6.3 technicians per hospital) and in the centre (6.2 technicians per hospital). The West also had the highest number of cases of pulmonary TB which may be the result of large workload in that area. There were more technicians per lab in TB-specific

\begin{tabular}{|c|c|c|c|c|c|}
\hline \multicolumn{6}{|l|}{ Smear } \\
\hline TB-specific hospitals with testing capacity & $165(81)$ & $177(87)$ & & & 198 (98) \\
\hline Overall test volume & 644217 & 973673 & & & 1710841 \\
\hline Tests per TB-specific hospital & 3904 & 5501 & & & 8641 \\
\hline TB-specific hospitals with testing capacity & $95(47)$ & $102(50)$ & & & 128 (63) \\
\hline Overall test volume & 57236 & 73934 & & & 161127 \\
\hline Tests per TB-specific hospital & 602 & 725 & & & 1259 \\
\hline \multicolumn{6}{|l|}{ Liquid culture } \\
\hline TB-specific hospitals with testing capacity & $20(10)$ & $44(22)$ & & & $62(31)$ \\
\hline Overall test volume & 27120 & 50464 & & & 114349 \\
\hline Tests per TB-specific hospital & 1356 & 1147 & & & 1844 \\
\hline \multicolumn{6}{|l|}{ Average lab technicians per TB-specific hospital } \\
\hline Central & & & & & 6.2 \\
\hline Eastern & & & & & 6.3 \\
\hline Western & & & & & 7.8 \\
\hline \multicolumn{6}{|l|}{ MDR } \\
\hline TB patients tested for DST & & & 30211 & 36670 & 49270 \\
\hline Drug-resistant TB patients ${ }^{\#}$ & & & 10229 & 12782 & 16674 \\
\hline MDR-TB patients diagnosed & & & 5366 & 6816 & 8769 \\
\hline
\end{tabular}

Data are presented as $n$ (\%) or n. TB: tuberculosis; MDR-TB: multi drug-resistant TB; DST: drug susceptibility test. "\#the number of drug-resistant TB cases include that of MDR-TB cases. 
hospitals than there were in public health system institutions, which had only roughly 5 lab technicians per lab at provincial level and 1-2 technicians per lab at prefectural level [12]. This is not surprising given that salaries are generally lower in the public health system.

The data on drug-resistant TB in tuberculosis-specific hospitals was only available for the period since 2007, after which drug-resistant TB was diagnosed in TB-specific hospitals using conventional DST. The overall number of these tests increased by $63.1 \%$ between 2007 and 2009 . The percentages of cases of drug-resistant TB and MDR-TB, among those tested for DST, stood at 33.9\% and $17.8 \%$ respectively, in $2007,34.9 \%$ and $18.6 \%$ respectively, in 2008 , and $33.8 \%$ and $17.8 \%$ respectively, in 2009 . There was no significant increase in the proportions of drug-resistant TB or MDR-TB among those tested for DST from 2007 to 2009 ( $\mathrm{p}>0.05)$.

A recent national survey on drug-resistant TB revealed that $8.3 \%$ of smear-positive TB patients tested for drug susceptibility had MDR TB in the public health system in 2007 [13]. In this study, we found the comparable statistic to be $17.8 \%$ in TB-specific hospitals in 2009 , which was about two times that in the public health system 2 years previously. This indicates the important role that is played by TB-specific hospitals in drug-resistant TB and MDR-TB treatment management. Previous studies have demonstrated that the abuse of anti-TB drugs, especially second-line drugs, is an ongoing area of concern in TB-specific hospitals $[14,15]$. Given the high level of prevalence of drug-resistant TB within tuberculosis-specific hospitals, it is imperative that moves are made to ensure a standardised treatment regimen for drug-resistant TB and MDR-TB patients across both systems. An integral element of this will be to strengthen follow-up processes within the tuberculosis-specific hospitals.

\title{
@ERSpublications
}

TB hospitals are critical for control of DR-TB, hence strengthened collaboration with public health systems is needed http://ow.ly/YnikY

\author{
Yu Pang ${ }^{1,2,3,4,7}$, Jian Du ${ }^{1,2,3,7}$, Zhi Zhen Qin ${ }^{5,6,7}$, Zoë Greenwald ${ }^{5}$, Yuhong Liu ${ }^{1,2,3}$, Fengling Mi ${ }^{1,2,3}$, Yanlin Zhao ${ }^{4}$ \\ and Liang $\mathrm{Li}^{1,2,3}$ \\ ${ }^{1}$ Beijing Chest Hospital, Capital Medical University, Beijing, China. ${ }^{2}$ Beijing Tuberculosis and Thoracic Tumor Research \\ Institute, Beijing, China. ${ }^{3}$ Administration Office, Clinical Center on Tuberculosis, Chinese Center for Disease Control \\ And Prevention, Beijing, China. ${ }^{4}$ National Center for Tuberculosis Control and Prevention, Chinese Center for Disease \\ Control and Prevention, Beijing, China. ${ }^{5}$ Dept of Epidemiology and Biostatistics, McGill University, Montreal, Canada. \\ ${ }^{6}$ Stop TB Partnership, Geneva, Switzerland. ${ }^{7}$ These authors contributed equally to this paper.
}

Correspondence: Liang Li, Beijing Chest Hospital, Capital Medical University, No. 97, Machang, Tongzhou District, Beijing, China. E-mail: liliang69@tb123.org

Received: June 182015 | Accepted after revision: Jan 192016 | First published online: Feb 252016

Support statement: This study was supported by the National Health and Family Planning Commission.

Conflict of interest: None declared.

Acknowledgements: We are grateful to all the local participants for their help throughout this survey. The authors are grateful to Jacob Creswell, Team Leader at TB REACH, Stop TB Partnerships, Geneva, Switzerland, for his critical review.

\section{References}

1 World Health Organization. Global Tuberculosis Report. Geneva, WHO Press, 2015. Available from www.who.int/ tb/publications/global_report/en/

2 Pang Y, Zhou Y, Zhao B, et al. Spoligotyping and drug resistance analysis of Mycobacterium tuberculosis strains from national survey in China. PLoS One 2012; 7: e32976.

3 World Health Organization. Global Update on the Health Sector Response to HIV, 2014. Geneva, WHO Press, 2014. Available from www.who.int/hiv/pub/progressreports/update2014/en/

4 World Health Organization. Strategic Approach for the Strengthening of Laboratory Services for Tuberculosis Control. Geneva, World Health Organization, 2006.

5 Paramasivan $\mathrm{CN}$, Lee E, Kao $\mathrm{K}$, et al. Experience establishing tuberculosis laboratory capacity in a developing country setting. Int J Tuberc Lung Dis 2010; 14: 59-64.

6 National Health and Family Planning Commission (NHFPC) of the People's Republic of China. China Health and Family Planning Statistics Yearbook. Beijing, Peking Union Medical College Press, 2013.

7 Wang L, Liu J, Chin DP. Progress in tuberculosis control and the evolving public-health system in China. Lancet 2007; 369: 691-696.

8 Ministry of Health of the People's Republic of China. National Tuberculosis Control Programme (2011-2015). Beijing, Ministry of Health, 2011.

9 Department of Disease Control, Department of Medical Administraton, Chinese Center for Disease Control and Prevention. Guidelines for Implementing the National Tuberculosis Control Program in China. Beijing, 2008.

10 Ministry of Health of the People's Republic of China. A List of Human Pathogenic Microorganisms. Beijing, Ministry of Health, 2006. 
11 Ministry of Health of the People's Republic of China. National Tuberculosis Control Programme (2006-2010). Beijing, Ministry of Health, 2006.

12 Chen S, Liu B, Wang X, et al. Analysis of human resource situation about TB control in Zhejiang. Chin J Antituberc 2006; 28: 345-347.

13 Zhao Y, et al. National survey of drug-resistant tuberculosis in China. N Engl J Med 2012; 366: 2161-2170.

$14 \mathrm{Li} \mathrm{W}$, Sun Q, Yan Y, et al. Investigation of the second line anti TB drugs usage in specialist hospitals and tuberculosis control institutions. Chin J Antituberc 2010; 32: 706-709.

15 Liu X, Wang L, Du Y, et al. Current status of case referring and treatment of tuberculosis inpatients in four tuberculosis specialist hospitals in China. Chin J Antituber 2010; 32: 775-778.

\title{
First independent evaluation of QuantiFERON-TB Plus performance
}

\author{
To the Editor:
}

Tuberculosis elimination requires an effective strategy to diagnose and treat people infected with Mycobacterium tuberculosis who would otherwise be at high risk of developing and transmitting active disease $[1,2]$. The diagnostic tools for latent tuberculosis infection (LTBI) are the tuberculin skin test (TST) and the T-cell interferon- $\gamma$ release assays (IGRAs). Two IGRAs are commercially available, QuantiFERON-TB Gold In-Tube (QFT-GIT) (Qiagen, Hilden, Germany) and T-SPOT.TB (Oxford Immunotec, Abingdon, UK). Compared to the TST, IGRAs offer operational advantages and higher specificity in the bacille Calmette-Guérin (BCG)-vaccinated population [3], and they are at least as sensitive for LTBI [4]. However, IGRAs have limitations: reduced sensitivity in children and immunocompromised subjects, including HIV-infected individuals [3, 4]; failure to discriminate between active tuberculosis and LTBI; and poor correlation with the risk of progression to active disease [3].

QuantiFERON-TB Plus (QFT-Plus) is a new generation of QTF-GIT [5] that includes an additional antigen tube (TB2). The TB1 tube contains ESAT-6- and CFP-10-derived peptides (TB7.7, present in QFT-GIT, has been removed), designed to elicit cell-mediated immune responses from $\mathrm{CD} 4{ }^{+} \mathrm{T}$-helper lymphocytes. TB2 contains newly designed, shorter peptides able to stimulate interferon- $\gamma$ production by both $\mathrm{CD}^{+}$and $\mathrm{CD}^{+}$T-cells. Previous studies have reported higher $M$. tuberculosis-specific CD8 ${ }^{+}$T-cell responses in those with active tuberculosis disease compared to LTBI [6] and in those with recent $M$. tuberculosis exposure [7]. In addition, $M$. tuberculosis-specific $\mathrm{CD}^{+}$T-cells producing interferon- $\gamma$ have been detected in active tuberculosis subjects with HIV co-infection [8] and in young children with TB disease [9].

IGRAs primarily serve as tests for LTBI in clinical practice and, to date, they have no place in active tuberculosis diagnosis [10]. In the absence of a gold-standard test for LTBI against which to compare test accuracy, sensitivity and specificity are estimated using surrogate reference standards. Sensitivity is estimated in confirmed tuberculosis cases, while specificity is estimated in low-risk individuals with no known tuberculosis exposure in low-incidence settings. From November 2014 to September 2015, 119 consecutive individuals with active tuberculosis and 106 low-risk controls were enrolled and tested with QFT-Plus at different sites in Italy (Villa Marelli-Niguarda Hospital and San Raffaele Hospital, Milan; INMI, Rome; Sant'Orsola-Malpighi University Hospital, Bologna; and San Matteo University Hospital, Pavia) and one in the UK (Royal Free Hospital, London). The active tuberculosis group included adult patients (aged $\geqslant 18$ years) with microbiologically confirmed tuberculosis (either by nucleic acid amplification or culture), who had received $<15$ days of anti-tuberculosis drugs. HIV co-infected individuals and immunocompromised patients were not excluded. Additional information was collected regarding: results of QFT-GIT, BCG vaccination status, and demographic, clinical and microbiological data. Healthy controls were recruited among students at the time of enrolment at the University Vita-Salute in Milan (low-risk population, non-BCG vaccinated); all subjects were interviewed and screened for the absence of any risk factors for M. tuberculosis exposure. The study was approved by the ethical committees of all the participating centres and informed consent was obtained from study subjects before blood sample collection.

QFT-Plus kits were donated by Qiagen and used according to the manufacturer's instructions [5]. Experiments were performed in three different laboratories (San Raffaele Hospital, Sant'Orsola-Malpighi 